\title{
Prediction and verification of auxin-ethylene crosstalk gene networks
}

Ubogoeva E. ${ }^{1,2 *}$, Levitsky V., ${ }^{1,2}$, Zemlyanskaya E. ${ }^{1,2}$

${ }^{1}$ Novosibirsk State University, Novosibirsk, Russia

${ }^{2}$ Institute of Cytology and Genetics, SB RAS, Novosibirsk, Russia

*e-mail:ubogoeva@gmail.com

Plant hormones auxin and ethylene are the key regulators of plant growth and development, which are widely used in agriculture. Crosstalk between these hormones is essential for the regulation of various physiological processes. Morphogenetic effects of ethylene are often provided by modulation of auxin biosynthesis and transport that cause changes in auxin distribution. The crosstalk is reciprocal since auxin induces ethylene biosynthesis. However, the molecular mechanisms underlying this crosstalk are still poorly understood. Here, we perform a genome-wide prediction of the molecular events of the auxin-ethylene crosstalk and their associations with certain biological processes, as well as investigate the underlying molecular mechanisms. We suggest an algorithm to predict the genes potentially involved in the crosstalk between two signaling pathways based on their functional annotation followed by transcription factor (TF) binding and expression pattern analyses. We applied the algorithm to identify auxin-sensitive genes that are associated with ethylene biosynthesis, signaling or ethylene response. As a result, we predicted over a hundred potential mediators for auxin-ethylene crosstalk, most of them were not reported to mediate auxin-ethylene crosstalk previously. Recruiting DAP-seq data, for some candidates we predicted potential mechanisms that could be utilized to regulate auxin-ethylene crosstalk. By analysis of expression patterns of predicted mediators and their putative regulators we made assumptions about the biological processes targeted by auxin-ethylene crosstalk. Some of the predictions were verified by qRT-PCR.

Acknowledgements: Supported by Russian Foundation for Basic Research and the government of Novosibirsk region, grant No. 18-44-540039. 